\title{
Comparison of Objective and Subjective Diagnostic Tests for Assessing Oral Dryness in Healthy Participants
}

\author{
Jun-Hee Shin, Hye-Kyoung Kim, Mee-Eun Kim \\ Department of Oral Medicine, Dankook University College of Dentistry, Cheonan, Korea
}

Received September 12, 2021

Revised October 5, 2021

Accepted October 5, 2021

Correspondence to:

Mee-Eun Kim

Department of Oral Medicine, Dankook

University College of Dentistry, 119 Dandae-

ro, Dongnam-gu, Cheonan 31116, Korea

Tel: +82-41-550-1915

Fax: +82-50-5434-7951

E-mail: meunkim@dankook.ac.kr

https://orcid.org/0000-0001-9332-532X
Purpose: Xerostomia is subjective feeling of dry mouth. It is complicated and multifactorial, which burdens clinicians in diagnosis and treatment of the problem. The goal of this study was to discuss the clinical importance of salivary flow rate, $\mathrm{pH}$ and subjective symptoms for evaluating oral dryness among young healthy male subjects.

Methods: Thirty male participants were recruited in this study (mean age \pm standard deviation of $25.70 \pm 1.84$ ). All participants completed 'Xerostomia Inventory' to measure subjective oral dryness scores. Unstimulated saliva and stimulated saliva were collected from each participant twice a day at 12:00 pm and 5:00 pm, using spitting method. Salivary flow rates and $\mathrm{pH}$ were measured immediately after collection. Relationship between objective and subjective measurements were analyzed.

Results: There were excellent intra-examiner reliability for salivary flow rate and $\mathrm{pH}$ and good internal consistency for Xerostomia Inventory. Objective measurements and subjective symptoms did not exhibit positive association. Salivary flow rate in unstimulated and stimulated condition showed positive association and also for salivary $\mathrm{pH}$. Stimulated salivary flow rate also presented positive correlation with stimulated salivary $\mathrm{pH}$.

Conclusions: Comprehensive assessment of objective measurements and subjective symptoms may be complimentary for assessing oral dryness, which would assist in implementing early interventions to improve patient's quality of life.

Key Words: Flow rate; pH; Questionnaire; Saliva; Xerostomia

\section{INTRODUCTION}

Saliva is essential for physical health. Without saliva, teeth are susceptible to rampant caries, abrasion, attrition, and erosion [1]. Oral mucosa is vulnerable to bacterial, viral, and fungal infection [1]. Food is hard to taste and digest, causing systemic malnutrition [1]. According to a recent report focusing on Oral Health Related Quality of Life (OHRQoL), not only physical but also psychological and social functioning are impaired [2]. Some patients experience choking moments in the middle of the night, disrupted of their sleep patterns [2]. Some are excluded from socialization because they have difficulty in speaking and eating normal food [2]. These devastating symptoms are what xerostomia patients suffer every day. About one in five people suffer from xerostomia worldwide [3]. Xerostomia is subjective feeling of dry mouth [4]. The prevalence is higher among older individuals, due to polypharmacy and onset of various medical conditions (e.g., Sjögren syndrome, head and neck radiation) [4]. Xerostomia and salivary gland hypofunction are different. Salivary gland hypofunction is a measurable reduction in salivary output [4]. The most advocated method for diagnosis of salivary gland hypofunction is measuring unstimulated and

Copyright (c) 2021 Korean Academy of Orofacial Pain and Oral Medicine.

(c) This is an open-access article distributed under the terms of the Creative Commons Attribution Non-Commercial License (http://creativecommons.org/licenses/by-nc/4.0/), which permits unrestricted non-commercial use, distribution, and reproduction in any medium, provided the original work is properly cited. 
stimulated whole salivary flow rates [4]. However, copious reports have suggested weak to no correlation between salivary flow rates and individuals' own subjective descriptions $[5,6]$. As xerostomia, subjective feeling of oral dryness, is complex and multifactorial, it is very challenging to assess clinically. Not only quantity but also quality such as molecular components ( $\mathrm{pH}$, viscosity, etc.) of saliva has gained attention in understanding the complexity of the condition [5]. Questionnaires have been developed to assess subjective feelings of dry mouth in numerical scales [2].

The aim of this study was to measure quantity and quality of saliva - whole salivary flow rates and $\mathrm{pH}$ - of young healthy adults and to analyze the relationship between the measurements. Using 'Xerostomia Inventory' (XI), this research also measured subjective oral dryness scores and compared them with objective measurements.

\section{MATERIALS AND METHODS}

\section{Subjects}

This research was performed at the Department of Orofacial Pain and Medicine in Dankook University Dental Hospital and was approved by the Institutional Review Board (IRB no. DKUDH IRB 2021-1-002). Thirty healthy males were recruited from January 26th to March 17th, 2021. All subjects voluntarily participated, and their medical history was collected through interview. They were all nonsmokers, without any systemic diseases or medication with xerogenic effects.

\section{Xerostomia Inventory}

XI was used to measure subjective oral dryness in a numerical scale (Table 1). The original English version was translated into Korean, by Korean experts fluent in English, through multiple revisions [7]. In a prospective cohort of 194 Korean patients with Sjögren syndrome, XI reliably reflected the degree of xerostomia [7]. Therefore, it was selected as the tool to estimate severity of xerostomia among healthy participants in our study. This questionnaire contains 11 items, each on a 5-point scale. The scores are summed ranging from 11 (no dry mouth) to 55 (extremely dry mouth). Some items refer directly to symptoms related to xerostomia $(2,4,8,9,10,11)$ and others refer to coping strategies $(1,3,6)$ as well as functioning limitations $(5,7)$ [2]. Before the salivary collection, all participants answered the questionnaire.

\section{Saliva Collection}

All participants received written instructions and education regarding the saliva collection. The subjects were refrained from drinking alcohol on the night before collection and eating or drinking except water for 90 minutes before each sampling [1]. Salivary samples were collected in restful and quiet circumstances [1]. The room temperature was managed by central heating system of Dankook Dental Hospital around $24^{\circ} \mathrm{C}$ to $25^{\circ} \mathrm{C}$. The subjects were instructed to sit comfortably resting for 2 minutes. Prior to collection they rinsed the mouth with water and emptied their mouth by an initial swallow [1]. Unstimulated saliva samples were collected for 5 minutes by spitting method in graded tubes (Fig. 1) [1,8]. Then, salivary glands were stimulated by chewing a paraffin pellet (Aurosan GmbH, Essen, Germany; Fig. 2) for 2 minutes [1,8]. After a swallow, stimulated saliva was also collected for 5 minutes [1,8]. Samples were collected twice a day around 12:00 pm and 5:00 pm. Four salivary samples were collected for each participant; unstimulated saliva, stimulated saliva at 12:00 pm and 5:00 pm. Total 120 salivary samples were collected from 30 patients. The salivary flow rates were measured right after collection by reading the scales on the tubes. For very foamy salivary

Table 1. Evaluation of xerostomia using self-reported questionnaire, Xerostomia Inventory

\begin{tabular}{lrc}
\hline \multicolumn{1}{c}{ Item } & Mean & SD \\
\hline 1. I sip liquids to aid in swallowing food. & 1.80 & 0.85 \\
2. My mouth feels dry when eating a meal. & 1.20 & 0.41 \\
3. I get up at night to drink. & 1.60 & 0.77 \\
4. My mouth feels dry. & 1.30 & 0.53 \\
5. I have difficulty in eating dry foods. & 1.70 & 0.88 \\
6. I suck sweets or cough lollies to relieve dry & 1.20 & 0.55 \\
$\quad$ mouth. & & \\
7. I have difficulties swallowing certain foods. & 1.13 & 0.43 \\
8. The skin of my face feels dry. & 2.10 & 0.96 \\
9. My eyes feel dry. & 2.30 & 1.12 \\
10. My lips feel dry. & 1.63 & 0.81 \\
11. The inside of my nose feels dry. & 2.13 & 0.94 \\
Total score & 18.10 & 4.82 \\
\hline
\end{tabular}

SD, standard deviation.

Data from the article of Thomson et al. (Community Dent Health 1999;16:12-17) [4]. 


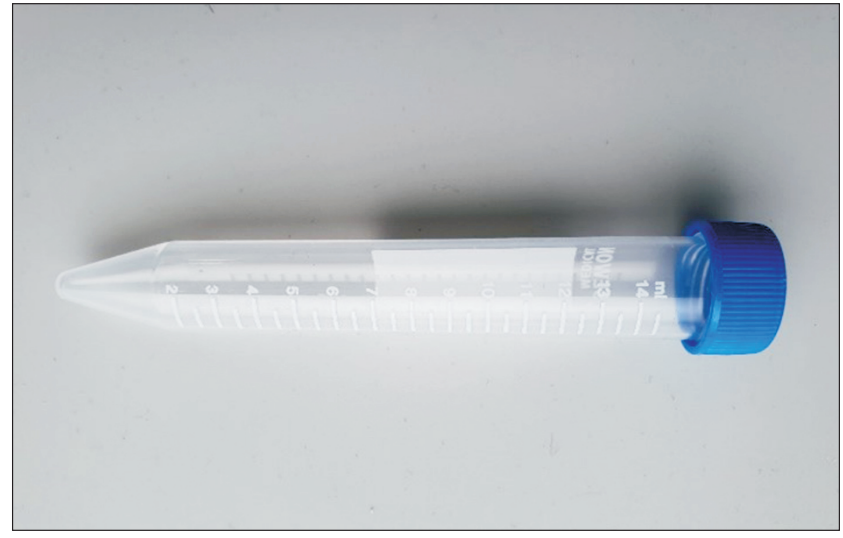

Fig. 1. Tube for measurement of salivary flow rate.

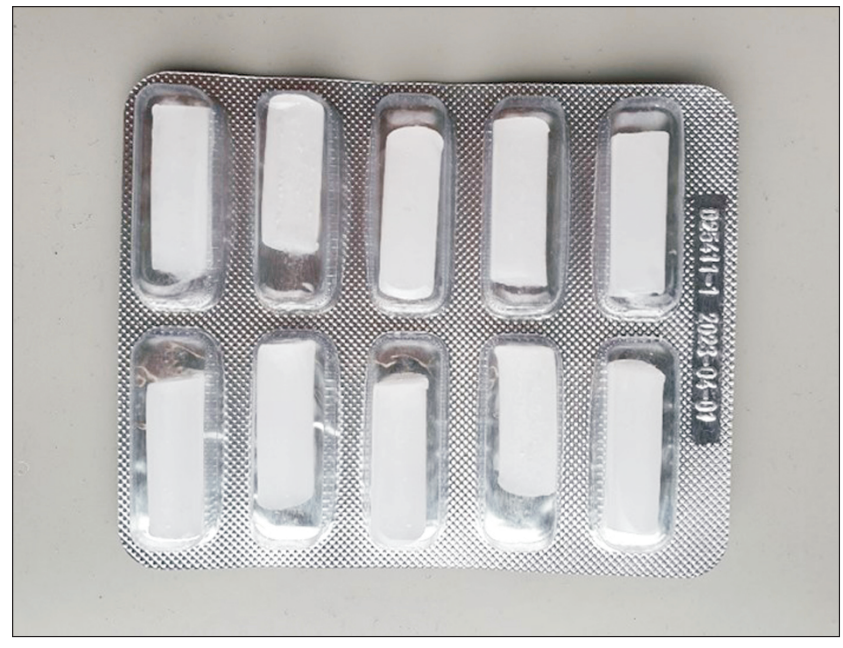

Fig. 2. Paraffin pellets for stimulation of saliva.

samples, top bubbles were excluded from the measurement and only the fluid below was measured from the scale of graded tube [9].

\section{4. $\mathrm{pH}$ Measurement}

A pH meter (LAQUA twin PH-33; Horiba, Kyoto, Japan; Fig. 3) was used to measure the $\mathrm{pH}$ of saliva. The $\mathrm{pH}$ meter was calibrated twice a day at 12:00 pm and 5:00 pm using buffers of pH 7 and pH 4.01. Immediately after collection, a small portion of saliva from the collection tube was poured into the $\mathrm{pH}$ sensor for measurement. After each measurement, the $\mathrm{pH}$ meter was washed with fresh tab water and the moisture was removed with soft tissue.

\section{Statistical Analysis}

Depending on the type and time of measurement, total

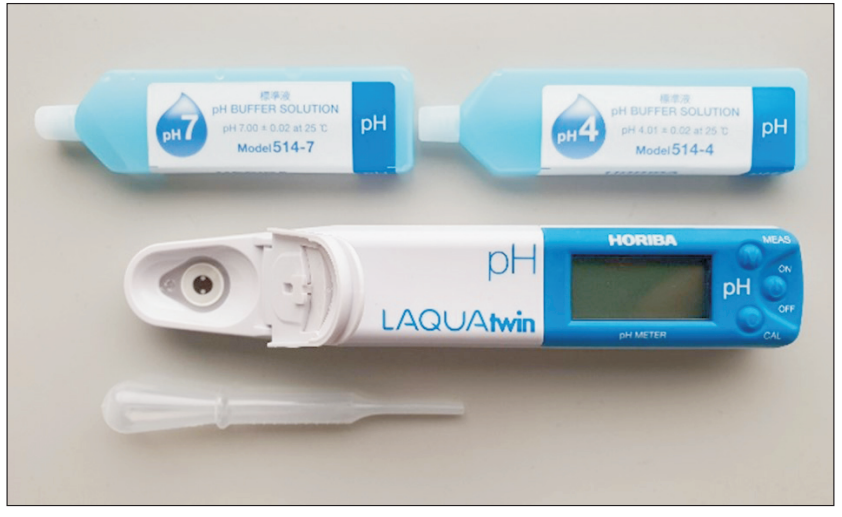

Fig. 3. $\mathrm{pH}$ meter for measurement of salivary $\mathrm{pH}$.

120 samples of saliva were divided into four groups; unstimulated saliva collected at 12:00 pm, stimulated saliva collected at 12:00 pm, unstimulated saliva collected at 5:00 pm, stimulated saliva collected at 5:00 pm.

Descriptive analysis was performed for calculating means and standard deviations (SDs) of questionnaires (the scores of each item and the total scores), salivary flow rates and $\mathrm{pH}$ of the four groups. For intra-examiner reliability between saliva measured at 12:00 pm and 5:00 pm, intraclass correlation coefficient (ICC) of salivary flow rate and $\mathrm{pH}$ was calculated.

To measure internal consistency of items in XI questionnaire, Cronbach alpha was calculated. Statistical differences of unstimulated and stimulated salivary flow rates were calculated using independent t-test, and also for unstimulated and stimulated salivary $\mathrm{pH}$. To estimate correlations between questionnaire, salivary flow rate and $\mathrm{pH}$, Pearson correlation analysis was used. IBM SPSS Statistics version 25.0 for window (IBM Corp., Armonk, NY, USA) was used for statistical analysis and significant level was set at $\mathrm{p}=0.05$.

\section{RESULTS}

\section{Subjects}

The mean age $\pm \mathrm{SD}$ of 30 healthy males aged from 23 to 30 years was $25.70 \pm 1.84$. A total number of 120 saliva samples were collected from 30 young healthy male participants in this study. 


\section{Internal Consistency of Xerostomia Inventory}

Table 1 shows the mean score of each item and the mean total score of 11 items (XI score) in 30 healthy participants. The mean XI score \pm SD was $18.1 \pm 4.82$. The XI questionnaire used in this study revealed good internal consistency (Cronbach alpha $=0.814$ ).

\section{Intra-examiner Reliability of Salivary Flow Rate}

Intra-examiner reliability of salivary flow rates measured by a single examiner (JHS) was calculated using the ICC (Table 2). The ICCs demonstrated excellent agreements in repeatability of the measurements (ICC $=0.931, p<0.001$ for unstimulated saliva; ICC $=0.952$, $\mathrm{p}<0.001$ for stimulated saliva). The mean scores \pm SD of unstimulated salivary flow rate were $0.62 \pm 0.32 \mathrm{~mL} / \mathrm{min}$ and $0.70 \pm 0.35 \mathrm{~mL} / \mathrm{min}$ at $12: 00$ $\mathrm{pm}$ and 5:00 pm, respectively. In case of stimulated salivary flow rate, the mean scores \pm SD were $1.71 \pm 0.64 \mathrm{~mL} / \mathrm{min}$, and $1.70 \pm 0.66 \mathrm{~mL} / \mathrm{min}$ at $12: 00 \mathrm{pm}$ and 5:00 pm, respectively. Stimulated salivary flow rate was significantly higher than unstimulated salivary flow rate at 12:00 pm and 5:00 pm $(\mathrm{p}<0.001$ for both).

\section{Intra-examiner Reliability of Salivary $\mathrm{pH}$}

Intra-examiner reliability of salivary $\mathrm{pH}$ measured by the examiner (JHS) was measured using the ICC (Table 3).
The ICCs demonstrated excellent agreements in repeatability of the measurements (ICC $=0.835, p<0.001$ for unstimulated saliva, ICC $=0.934, \mathrm{p}<0.001$ for stimulated saliva). The mean \pm SD scores of unstimulated salivary $\mathrm{pH}$ were $7.07 \pm 0.28$ and $7.17 \pm 0.24$ at 12:00 pm and 5:00 pm, respectively. The mean \pm SD scores of stimulated salivary $\mathrm{pH}$ were $7.51 \pm 0.29$ and $7.59 \pm 0.27$ at 12:00 pm and 5:00 pm, respectively. Stimulated salivary $\mathrm{pH}$ was significantly higher than unstimulated salivary flow rate at 12:00 pm and 5:00 pm ( $p<0.001$ for both).

\section{Correlations between Xerostomia Symptoms, Salivary Flow Rate and pH}

Multiple comparisons using Pearson correlation analysis were performed to investigate correlations between the total score of xerostomia questionnaire, salivary flow rate and salivary $\mathrm{pH}$ in healthy participants (Table 4). There were no significant associations between subjective symptoms and measurements including salivary flow rate $(\mathrm{r}=-0.245$, $p=0.192$ for unstimulated saliva; $r=-0.200, p=0.290$ for stimulated saliva) and salivary $\mathrm{pH}(\mathrm{r}=-0.057, \mathrm{p}=0.764$ for unstimulated saliva; $r=-0.200, p=0.289$ for stimulated saliva). On the other hand, unstimulated salivary flow rate presented strong positive correlation with stimulated salivary flow rate $(r=0.688, p<0.001)$. Like salivary flow rate,

Table 2. Intra-examiner reliability of salivary flow rate

\begin{tabular}{lcccc}
\hline Salivary flow rate & Test & Retest & ICC & p-value $^{\text {a }}$ \\
\hline Unstimulated $(\mathrm{mL} / \mathrm{min})$ & $0.62 \pm 0.32$ & $0.70 \pm 0.35$ & 0.931 & $<0.001$ \\
Stimulated $(\mathrm{mL} / \mathrm{min})$ & $1.71 \pm 0.64$ & $1.70 \pm 0.66$ & 0.952 & $<0.001$ \\
p-value $^{\text {b }}$ & $<0.001$ & $<0.001$ & & \\
\hline
\end{tabular}

ICC, intraclass correlation coefficient.

Values are presented as mean \pm standard deviation. Test: measurement at 12:00 pm, Retest: measurement at 5:00 pm.

${ }^{a}$ Statistical significance from computing ICC.

${ }^{\mathrm{b}}$ Statistical significance from independent t-test.

Table 3. Intra-examiner reliability of salivary $\mathrm{pH}$

\begin{tabular}{ccccc}
\hline Salivary $\mathrm{pH}$ & Test & Retest & ICC & $\mathrm{p}^{- \text {value }}$ \\
\hline Unstimulated $(\mathrm{pH})$ & $7.07 \pm 0.28$ & $7.17 \pm 0.24$ & 0.835 & $<0.001$ \\
Stimulated $(\mathrm{pH})$ & $7.51 \pm 0.29$ & $7.59 \pm 0.27$ & 0.934 & $<0.001$ \\
p-value $^{\mathrm{b}}$ & $<0.001$ & $<0.001$ & & \\
\hline
\end{tabular}

ICC, intraclass correlation coefficient.

Values are presented as mean \pm standard deviation. Test: measurement at 12:00 pm, Retest: measurement at 5:00 pm.

${ }^{a}$ Statistical significance from computing ICC.

${ }^{\mathrm{b}} \mathrm{S}$ tatistical significance from independent t-test. 
Table 4. Correlations between subjective symptoms, salivary flow rate and salivary $\mathrm{pH}$

\begin{tabular}{|c|c|c|c|c|c|}
\hline Pearson correlation analysis & $\begin{array}{l}\text { Xerostomia } \\
\text { symptom }\end{array}$ & USS (mL/min) & $\mathrm{SS}(\mathrm{mL} / \mathrm{min})$ & USS (pH) & SS (pH) \\
\hline Xerostomia symptom & 1 & $-0.245(0.192)$ & $-0.200(0.290)$ & $-0.057(0.764)$ & $-0.200(0.289)$ \\
\hline USS (mL/min) & & 1 & $0.688\left(<0.001^{* *}\right)$ & $0.131(0.491)$ & $0.205(0.278)$ \\
\hline $\mathrm{SS}$ (mL/min) & & & 1 & $0.148(0.434)$ & $0.382(0.037 *)$ \\
\hline USS (pH) & & & & 1 & $0.746(<0.001 * *)$ \\
\hline $\mathrm{SS}(\mathrm{pH})$ & & & & & 1 \\
\hline
\end{tabular}

USS, unstimulated salivary flow rate; SS, stimulated salivary flow rate.

Values are presented as correlation coefficient ( $p$-value).

Correlation is significant at ${ }^{*} p<0.05,{ }^{* *} p<0.01$ (2-tailed).

unstimulated salivary $\mathrm{pH}$ revealed significantly strong positive correlation with stimulated salivary $\mathrm{pH}(\mathrm{r}=0.746$, $\mathrm{p}<0.001)$. Unlike the correlation between unstimulated salivary flow rate and unstimulated salivary $\mathrm{pH}(\mathrm{r}=0.131$, $r=0.491$ ), there was moderate positive association between stimulated salivary flow rate and stimulated salivary $\mathrm{pH}$ $(r=0.382, p=0.037)$.

\section{DISCUSSION}

This investigation of normative data for objective measurements and subjective symptoms in healthy young males demonstrated excellent intra-examiner reliability for salivary flow rate and $\mathrm{pH}$ measurement and good internal consistency for XI. Interestingly, objective measurements and subjective symptoms did not exhibit positive association. On the other hand, unstimulated salivary flow rate and $\mathrm{pH}$ showed positive associations with stimulated salivary flow rate and $\mathrm{pH}$, respectively. Stimulated salivary flow rate also presented positive correlation with stimulated salivary $\mathrm{pH}$.

\section{Xerostomia Symptoms in Healthy Participants}

XI was developed to estimate severity of subjective symptoms in a continuous scale [4]. It has been used to evaluate OHRQoL in previous research and revealed good construct validity, internal consistency, and reliability $[7,10]$. In accordance with previous studies, this research also revealed good internal consistency (Cronbach alpha $=0.814$ ). In the paper of Thomson et al. [4], to the standard question 'how often does your mouth feel dry?', subjects who answered 'never' had the mean total score of 16.97 and those who answered 'occasionally' had 19.49. In another paper by Mizutani et al. [11], young adults (aged 18-24) who answered 'never' had the mean score of $18.9 \pm 6.9$ and who answered 'occasionally' scored $22.9 \pm 6.5$. The healthy subjects of this study hadn't experienced xerostomia before, and the average XI score was $18.10 \pm 4.82$, which is similar to previous studies. The items of high scores were 'my eyes feel dry', 'the inside of my nose feels dry' and 'the skin of my face feels dry' This may be because most of the subjects were dental students working with dental materials. The items of low scores were 'I have difficulties swallowing certain foods', 'my mouth feels dry when eating a meal' and 'I suck sweets or cough lollies to relieve my dry mouth' - the low scored questions were all related to eating. According to Löfgren et al. [5], perceived oral dryness associated with eating was highly indicative of salivary performance. This explains the low scores of questions related to eating among the healthy participants of our study. In actual xerostomic patients, whether they feel oral dryness associated with eating could indicate their decreased salivary performance.

\section{Salivary Flow Rate in Healthy Participants}

There was a wide range of salivary flow rates among the healthy individuals - unstimulated flow rate (mean $0.62 \pm 0.32 \mathrm{~mL} / \mathrm{min}$ and $0.70 \pm 0.35 \mathrm{~mL} / \mathrm{min}$ at $12: 00 \mathrm{pm}$ and 5:00 pm) and stimulated flow rate $(1.71 \pm 0.64 \mathrm{~mL} / \mathrm{min}$, and $1.70 \pm 0.66 \mathrm{~mL} / \mathrm{min}$ at 12:00 pm and 5:00 pm). When measuring salivary flow rates repeatedly, circadian rhythms of salivary flow rates should be taken into consideration. In this research, there were excellent agreements in repeatability of measurements at 12:00 pm and 5:00 pm. Dawes reported circadian rhythms in the unstimulated salivary flow rates, which resembled sinusoidal pattern; lowest during the sleep around 3:00 am and highest at noon around 3:00 pm 
[12]. It is advisable to measure salivary flow rates at fixed time of the day to keep track of one's salivary function over time [1].

The stimulated salivary flow rate was significantly higher than unstimulated salivary flow rate and they were positively correlated. Salivary secretion is depended on cholinergic signaling from parasympathetic nerves [13]. Taste, olfaction, and mastication cause afferent signals, which are modified by central nervous system [13]. Efferent signals are delivered to salivary glands in autonomic nerves, which leads to increase in salivation [13]. In a study with actual dry mouth patients, flow rates of unstimulated and stimulated saliva were lower than our results, $0.05 \pm 0.05$ and $0.34 \pm 0.32$, and they were also positively correlated [14]. Considering the mechanism of salivary secretion, stimulation of salivary gland by gustatory or masticatory stimuli is recommended for patients with hyposalivation [1].

\section{Salivary $\mathrm{pH}$ in Healthy Participants}

In this experiment, the unstimulated salivary $\mathrm{pH}$ was $7.07 \pm 0.28$ and $7.17 \pm 0.24$ at $12: 00 \mathrm{pm}$ and $5: 00 \mathrm{pm}$, and the stimulated salivary $\mathrm{pH}$ was $7.51 \pm 0.29$ and $7.59 \pm 0.27$ at 12:00 pm and 5:00 pm. Like the salivary flow rate, circadian rhythms of salivary $\mathrm{pH}$ should be considered. In this research, there was excellent repeatability in the measurements at 12:00 pm and 5:00 pm. Choi et al. [15] illustrated circadian rhythms in the unstimulated salivary $\mathrm{pH}$, which was lowest during the sleep around 4:00 am and highest around 4:00 pm. It is recommended to measure salivary $\mathrm{pH}$ at fixed time of the day to record one's salivary function periodically [1]. The experiment revealed higher $\mathrm{pH}$ in stimulated saliva than unstimulated saliva and they were also positively correlated. This may be due to bicarbonate buffer system of saliva. Under normal condition, the $\mathrm{pH}$ of unstimulated saliva is about neutral [13]. Upon stimulation, not only the salivary flow rate but also the concentration of bicarbonate increases, resulting in higher $\mathrm{pH}$ [13]. Since the bicarbonate concentration of stimulated saliva is much higher than that of unstimulated saliva, it is likely to be controlled by autonomic nerve-mediated stimuli [13]. This may also explain moderate positive association between stimulated salivary flow rate and stimulated salivary $\mathrm{pH}$ $(\mathrm{r}=0.382, \mathrm{p}=0.037)$. In dry mouth patients, low salivary flow rate results in decreased intraoral $\mathrm{pH}$ due to reduced buffering capacity and acid clearance [15]. There is few research that studied both unstimulated and stimulated salivary $\mathrm{pH}$ in xerostomic patients, and even fewer studies that correlated them, which suggests further studies.

\section{Comparison of Xerostomia Symptoms and Measurements}

First, there were no significant associations between subjective symptoms and salivary flow rate. The most prominent criteria for hyposalivation are $0.1 \mathrm{~mL} / \mathrm{min}$ for unstimulated saliva and $0.7 \mathrm{~mL} / \mathrm{min}$ for stimulated whole saliva [6]. Unstimulated salivary flow rate is known to be more sensitive to dry mouth complaints, but stimulated saliva was more associated with oral dryness on daily life and severity of symptoms [14]. It is necessary to measure flow rates in both unstimulated and stimulated condition to assess xerostomia comprehensively [1]. Copious research has suggested low correlation between measures of salivary flow rates and individuals' own subjective description $[5,6]$. Salivary flow rates can be reduced as much as 50\% before the sensation of oral dryness occurs, but dry mouth can also be experienced in subjects with normal range of salivary flow rate $[5,6]$. According to Ship et al. [16], it is difficult to set standards of 'normal flow rate' but changes of salivary function over time has significant impact on oral health. XI scores are used to assess subjective change of symptoms and a change in XI score of 6 or more points is clinically meaningful [17]. Wiener et al. [18], has suggested that self-reported information, clinical examination, and routine salivary flow rates can increase early detection of oral dryness, which would assist in implementing early interventions to improve patient's quality of life.

Second, there were no significant associations between subjective symptoms and salivary $\mathrm{pH}$. There was scanty literature related to salivary $\mathrm{pH}$ and symptoms of oral dryness. One study of nasopharyngeal carcinoma patients who underwent radiotherapy, unstimulated salivary $\mathrm{pH}$ decreased, and xerostomia scores increased at 2 years follow up, but the correlation was not examined [19]. Further studies are necessary that correlates subjective symptoms and salivary $\mathrm{pH}$. In a systemic review of methods to diagnose subjective oral dryness and salivary gland function, evidence of efficacy of clinical methods to assess oral dryness 
is sparse [5]. There is a need for effective diagnostic criteria and functional tests to detect patients who may require treatment.

This study had several study limitations. Only male subjects were recruited to reduce variables among healthy participants in this study. Women undergo menstrual cycle periodically, and this is known to change volume and composition of saliva [20]. Another research revealed that women showed lower salivary flow rates compared to men, whereas salivary $\mathrm{pH}$ didn't show significant difference [21]. Inoue et al. [22] suggested lower unstimulated salivary flow rate among female due to smaller gland sizes. Li-Hui et al. [23] also demonstrated gender differences in salivary flow rate and biochemistry among young healthy subjects. On the other hand, Govindaraj et al. [24] revealed no significant difference among gender of salivary flow rate and $\mathrm{pH}$, although mean scores were lower in women. Future studies are needed to reveal salivary characteristics of different sexs.

According to Proctor and Carpenter [13], unstimulated salivary flow rate is misnomer since salivary secretion of fluid is only unstimulated in a complete absence of neural activation, which does not apply in the conscious subject with an intact innervation. To supplement, subjects in this study were thoroughly educated to refrain from moving their mouth during the collection of unstimulated saliva. The examiner (JHS) supervised the process in a fair distance to avoid disturbing the patients.

Salivary flow rate and $\mathrm{pH}$ are most accurate when measured right after collection. However, with multiple salivary samples in a day, it was impossible to calculate the measurements immediately. To reduce the errors, all samples were measured within 30 minutes after collection.

Although XI covers symptoms of xerostomia, coping strategies, and functional limitations, it does not cover the full range of impacts from xerostomia [2]. More comprehensive questionnaires that include not only physical impacts, but also psychological and social impacts are necessary to fully analyze diverse aspects of xerostomia.

Despite the limitations, this study evaluated xerostomia in a comprehensive way, from quantitative assessment of salivary flow rate, qualitative measurement of salivary $\mathrm{pH}$ to subjective assessment using the questionnaire in healthy participants. The research also provided clinical evidence for absence of association between the objective measurements and subjective symptoms in healthy participants, which has not been studied previously, especially regarding salivary $\mathrm{pH}$.

In conclusion, comprehensive assessment of objective measurements and subjective symptoms may be complimentary for assessing oral dryness, which would assist in implementing early interventions to improve patient's quality of life.

\section{CONFLICT OF INTEREST}

No potential conflict of interest relevant to this article was reported.

\section{ORCID}

\author{
Jun-Hee Shin \\ https://orcid.org/0000-0002-8306-1460 \\ Hye-Kyoung Kim \\ https://orcid.org/0000-0002-0734-5533 \\ Mee-Eun Kim \\ https://orcid.org/0000-0001-9332-532X
}

\section{REFERENCES}

1. Wong D. Salivary diagnostics. Ames: Wiley-Blackwell; 2008. pp. 27-59.

2. Gibson B, Periyakaruppiah K, Thornhill MH, Baker SR, Robinson PG. Measuring the symptomatic, physical, emotional and social impacts of dry mouth: a qualitative study. Gerodontology 2020;37:132-142.

3. Agostini BA, Cericato GO, Silveira ERD, et al. How common is dry mouth? Systematic review and meta-regression analysis of prevalence estimates. Braz Dent J 2018;29:606-618.

4. Thomson WM, Chalmers JM, Spencer AJ, Williams SM. The Xerostomia Inventory: a multi-item approach to measuring dry mouth. Community Dent Health 1999;16:12-17.

5. Löfgren CD, Wickström C, Sonesson M, Lagunas PT, Christersson C. A systematic review of methods to diagnose oral dryness and salivary gland function. BMC Oral Health 2012;12:29.

6. Sreebny LM, Valdini A. Xerostomia. Part I: relationship to other oral symptoms and salivary gland hypofunction. Oral Surg Oral Med Oral Pathol 1988;66:451-458.

7. Lee J, Koh JH, Kwok SK, Park SH. Translation and validation of a Korean version of the Xerostomia Inventory in patients with primary Sjögren's syndrome. J Korean Med Sci 2016;31:724-728.

8. Song CW, Kim HK, Kim ME. Clinical usefulness of $\mathrm{pH}$ papers in 
the measurement of salivary pH. J Oral Med Pain 2015;40:124129.

9. Alves C, Brandão M, Andion J, Menezes R. Use of graduated syringes for measuring salivary flow rate: a pilot study. Braz Dent $\mathrm{J}$ 2010;21:401-404.

10. Locker D. Dental status, xerostomia and the oral health-related quality of life of an elderly institutionalized population. Spec Care Dentist 2003;23:86-93.

11. Mizutani S, Ekuni D, Tomofuji T, et al. Relationship between xerostomia and gingival condition in young adults. J Periodontal Res 2015;50:74-79.

12. Dawes C. Circadian rhythms in human salivary flow rate and composition. J Physiol 1972;220:529-545.

13. Proctor GB, Carpenter GH. Salivary secretion: mechanism and neural regulation. Monogr Oral Sci 2014;24:14-29.

14. Suh KI, Lee JY, Chung JW, Kim YK, Kho HS. Relationship between salivary flow rate and clinical symptoms and behaviours in patients with dry mouth. J Oral Rehabil 2007;34:739-744.

15. Choi JE, Lyons KM, Kieser JA, Waddell NJ. Diurnal variation of intraoral pH and temperature. BDJ Open 2017;3:17015.

16. Ship JA, Fox PC, Baum BJ. How much saliva is enough? 'Normal' function defined. J Am Dent Assoc 1991;122:63-69.

17. Thomson WM. Measuring change in dry-mouth symptoms over time using the Xerostomia Inventory. Gerodontology 2007;24:30-
35.

18. Wiener RC, $\mathrm{Wu} B$, Crout R, et al. Hyposalivation and xerostomia in dentate older adults. J Am Dent Assoc 2010;141:279-284.

19. Sim C, Soong YL, Pang E, et al. Xerostomia, salivary characteristics and gland volumes following intensity-modulated radiotherapy for nasopharyngeal carcinoma: a two-year follow up. Aust Dent J 2018;63:217-223.

20. Kitamura N, Ohara I. Daily fluctuation of saliva secretion in women during the menstrual cycle. J Jpn Soc Nutr Food Sci 2010;63:79-85.

21. Fenoll-Palomares C, Muñoz Montagud JV, Sanchiz V, et al. Unstimulated salivary flow rate, $\mathrm{pH}$ and buffer capacity of saliva in healthy volunteers. Rev Esp Enferm Dig 2004;96:773-783.

22. Inoue $\mathrm{H}$, Ono K, Masuda W, et al. Gender difference in unstimulated whole saliva flow rate and salivary gland sizes. Arch Oral Biol 2006;51:1055-1060.

23. Li-Hui W, Chuan-Quan L, Long Y, Ru-Liu L, Long-Hui C, WeiWen C. Gender differences in the saliva of young healthy subjects before and after citric acid stimulation. Clin Chim Acta 2016;460:142-145.

24. Govindaraj S, Daniel M, Vasudevan S, Kumaran J. Changes in salivary flow rate, $\mathrm{pH}$, and viscosity among working men and women. Dent Med Res 2019;7:56-59. 